\title{
Incorporating Ocean Wave Spectrum Information in Short-Term Free-Surface Elevation Forecasting
}

\author{
Alexis Mérigaud ${ }^{\circledR}$ and John V. Ringwood, Senior Member, IEEE
}

\begin{abstract}
Real-time prediction of free-surface elevation or wave excitation force is necessary for a variety of applications, including noncausal optimal control of wave energy converters and detection of quiescent periods for offshore operations. Prediction methods can use past measurements at the point of interest, treating the wave elevation as a time series (looking backward). Alternatively, wave elevation values can be recorded at a set of locations about the point of interest and propagated in time and space through physical or statistical models (looking forward). In this paper, assuming Gaussian waves, a unified framework is proposed, which treats any combination of wave elevation values at various points in time and space as a Gaussian vector and covers both "backward" and "forward" approaches. It is shown that, using any given combination of measurement points in time and space, the optimal predictor, in a least mean square sense, is linear for any time horizon and can be directly derived from the wave spectrum, provided that the latter is known. The associated error can be readily calculated, thus providing, based on the wave spectrum, an upper bound on the predictability of the wave elevation process. In addition, the applicability of the spectrum-based predictor to real-time wave elevation forecasting is discussed.
\end{abstract}

Index Terms-Deterministic sea wave prediction, Gaussian process, linear waves, real-time wave prediction, wave spectrum.

\section{INTRODUCTION}

$\mathbf{R}$ EAL-TIME prediction of sea surface elevation has received significant interest in the past 20 years [1]. Possible applications include the detection of quiescent periods for safe marine operations [2], [3] and real-time optimal control of wave energy converters (WECs) to improve power capture [4]-[6].

Two main types of approaches for short-term wave forecasting are generally identified [7]: methods based on spatial prediction of wave elevation, through physical or stochastic propagation models, using one or more observations in the vicinity of the point of interest ("looking forward," following the terminology used in [2]), and methods using only past measurements at the point of interest; thus treating the wave elevation as a time series ("looking backward," following [2]). While the authors believe that the broad terminology of "forward/backward" is useful and consistent with the previous work, the "forward" label can re-

Manuscript received July 28, 2017; revised November 20, 2017; accepted March 28, 2018. This work was supported by the Science Foundation Ireland under Grant 12/RC/2302 for the Marine Renewable Ireland Centre. (Corresponding author: Alexis Mérigaud.)

Associate Editor: M. Haller.

The authors are with the Centre for Ocean Energy Research, Department of Electronic Engineering, Maynooth University, Maynooth, Ireland (e-mail: alexis.merigaud.2015@mumail.ie; john.ringwood@eeng.nuim.ie).

Digital Object Identifier 10.1109/JOE.2018.2822498 fer to both up-wave and down-wave measurements-though, as will become apparent, up-wave data are significantly more useful than down-wave data.

Typically, in the "looking forward" approach, signal processing tools are employed to identify a set of wave components with various frequencies, phases, and directions [1]. The identified components are subsequently propagated in space and time using physical propagation equations. Therefore, such methods are often termed deterministic sea wave prediction (DSWP), as opposed to the traditional stochastic description of ocean waves [8]. Accurate forecasts $(\geq 80 \%)$ can typically be achieved for prediction horizons of the order of $30 \mathrm{~s}$ [1], [9].

"Looking backward" techniques use measured data at the point of interest, to identify, as a background task, the parameters of a time-series model. The real-time prediction is performed by propagating the model in time. Although accurate predictions up to a few tens of seconds are reported in [7], those were obtained after offline filtering of the wave data, and thus it is yet unclear what can be achieved in real time by using a "looking backward" perspective. Time-series approaches, such as autoregressive (AR) models, have the advantage of simplicity, and naturally provide an interesting probabilistic framework to handle forecast uncertainties.

Deep-water ocean waves can be, in the vast majority of cases, modeled accurately as a linear, Gaussian stochastic process [8], which is entirely characterized by its energy spectrum [spectral density function (SDF)] or, alternatively, by its autocovariance function (ACVF). The Gaussian spectral representation characterizes the statistical properties of the wave elevation time series. Therefore, as highlighted in [6]

it may be expected that the ability to predict the excitation force, by using a stochastic model, is related to properties of the sea state (peak frequency and bandwidth) [...].

Furthermore, under the assumption of a stationary Gaussian sea, all the statistical information, which characterizes the wave elevation process, is contained within the wave SDF.

In this paper, the implications of the spectral wave representation, in terms of short-term wave forecasting, are investigated in detail. Treating measured and predicted wave elevation values as a Gaussian vector, a unified framework is proposed, which encompasses both "looking forward" and "looking backward" approaches. The proposed methodology relies on the linearity and stationarity assumptions, within the temporal and spatial scales considered. In particular, breaking, and rogue, waves are excluded from the analysis. Using any combination of mea- 
surement points in time and space, it is shown that the optimal predictor, in a mean square error sense and for any time horizon, is linear, and that its coefficients can be derived directly from the wave spectrum, provided that the latter is known. The error associated with the optimal predictor is also easily provided. The following two significant consequences are detailed in this study.

1) First, under the assumption of stationary Gaussian waves, any other predictor using the same combination of measurement points (in space and time) is suboptimal, with respect to the prediction law derived in this paper. For any given combination of measurement points in time and space, the spectrum-based predictor thus provides a theoretical estimate of the wave elevation process predictability.

2) Second, from a practical point of view, assuming that the wave elevation process is locally stationary, it could be possible to use spectral information to improve the forecasting process. Thus, instead of propagating physical equations in time and space (which can be computationally costly), or instead of identifying time-series models from measured data, the focus could be shifted toward estimating, in real time, the underlying wave spectrum, possibly using a combination of local measurements and outputs from real-time meteorological models. The optimal predictor would then be directly calculated from the spectrum, as in this paper. Overall, the short-term forecasting problem would be reduced to determine the wave SDF or ACVF, which reflect the physical processes underlying the wave dynamics, and therefore evolve on a relatively slow time scale, and present some degree of predictability using information from physical wave models used by meteorological agencies. ${ }^{1}$

Section II is a reminder of the Gaussian description of ocean waves, and illustrates, through the wave covariance function, how measurements at different locations contain usable statistical information. The optimal, spectrum-based predictor is given in Section III, for any combination of measurements taken at various spatial locations and points in the past. The following sections provide various numerical illustrations of the spectrum-based predictor performance: Waves measured solely at the point of interest ("looking backward" approach) is the subject of Section IV, whereas spatially distributed measurements ("looking forward") are considered in Section V for unidirectional wave fields, and Section VI considered directional wave fields. Finally, conclusions and discussions are presented in Section VII.

\section{GAUSSIAN DESCRIPTION OF OCEAN WAVES}

\section{A. Gaussian Waves}

Ocean waves can be modeled as a zero-mean Gaussian field in most conditions, i.e., as long as water depth is sufficient and the wave condition is not too extreme [8]. Let us consider a small duration with respect to the typical rate at which the sea con-

\footnotetext{
${ }^{1}$ See, for example, the European Centre for Medium-Range Weather Forecasts website https://www.ecmwf.int
}

dition evolves in time, and a relatively small area, with respect to the typical distance over which the sea condition evolves in space. Then, the wave elevation process can be considered as being stationary, which, for a Gaussian process, reduces to time invariance of the first- and second-order statistical moments [8], and it can be considered to be homogeneous, i.e., its statistical properties do not change over horizontal dimensions. Finally, the sea surface Gaussian process is also considered to be ergodic [8], which means that time averages of the process are equal to ensemble averages.

Under such conditions, the wave statistical properties are entirely characterized by the wave elevation spectrum [8] or, equivalently, by the wave ACVF. If the wave elevation at only one specific point is considered, (as in a "looking backward" approach), the wave elevation process is described as 1-D Gaussian stochastic process. If several points in space have to be considered, the space-time statistical properties of the process must be characterized.

\section{B. Wave Elevation as a Point Gaussian Process}

In the case of point measurements, the Gaussian wave field reduces to a 1-D Gaussian process. Such a stationary, ergodic Gaussian process is entirely characterized by its mean $(\bar{\eta}=0)$ and its $\mathrm{ACVF} R_{\eta \eta}$ is defined as follows:

$$
R_{\eta \eta}(\tau)=\lim _{T \rightarrow \infty} \frac{1}{2 T} \int_{-T}^{T} \eta(t) \eta(t+\tau) d t .
$$

The fact that $R_{\eta \eta}$ only depends on $\tau$ stems from the process stationarity. Furthermore, $R_{\eta \eta}(\tau)$ is an even function that is maximum at $\tau=0$, with $R_{\eta \eta}(0)$ representing the process variance.

Due to the ergodicity property of the process, $R_{\eta \eta}$ can also be defined as follows:

$$
R_{\eta \eta}(\tau)=\mathbb{E}[\eta(t) \eta(t+\tau)] .
$$

In addition, let us define the $\operatorname{SDF} S_{\eta \eta}(f)$ of the process as follows:

$$
S_{\eta \eta}(f)=\lim _{T \rightarrow \infty} \frac{1}{T}\left|H_{T}(f)\right|^{2}
$$

where $H_{T}(f)=\int_{-T}^{T} \eta(t) \mathrm{e}^{-i 2 \pi f t} d t$.

The ACVF and the SDF of the weakly stationary sea surface elevation process are a Fourier transform pair, according to the Wiener-Khintchine theorem. As a consequence, the statistical properties of the stationary, zero-mean Gaussian wave elevation process are entirely described by its ACVF, or equivalently by its SDF—-see [8] for more details.

In practice, the SDF is more often available than the ACVF: First, the wave spectrum can be easily estimated using Fourier transforms of a recorded wave elevation signal. Second, a sea state representation through an SDF is very informative since it explicitly shows the wave frequency content, which is useful in studying the mechanical response of ships or offshore structures. Finally, meteorological models that include ocean wave conditions also describe sea states by means of the wave SDF since wave generation and propagation models describe the evolution of wave energy at different frequencies. 

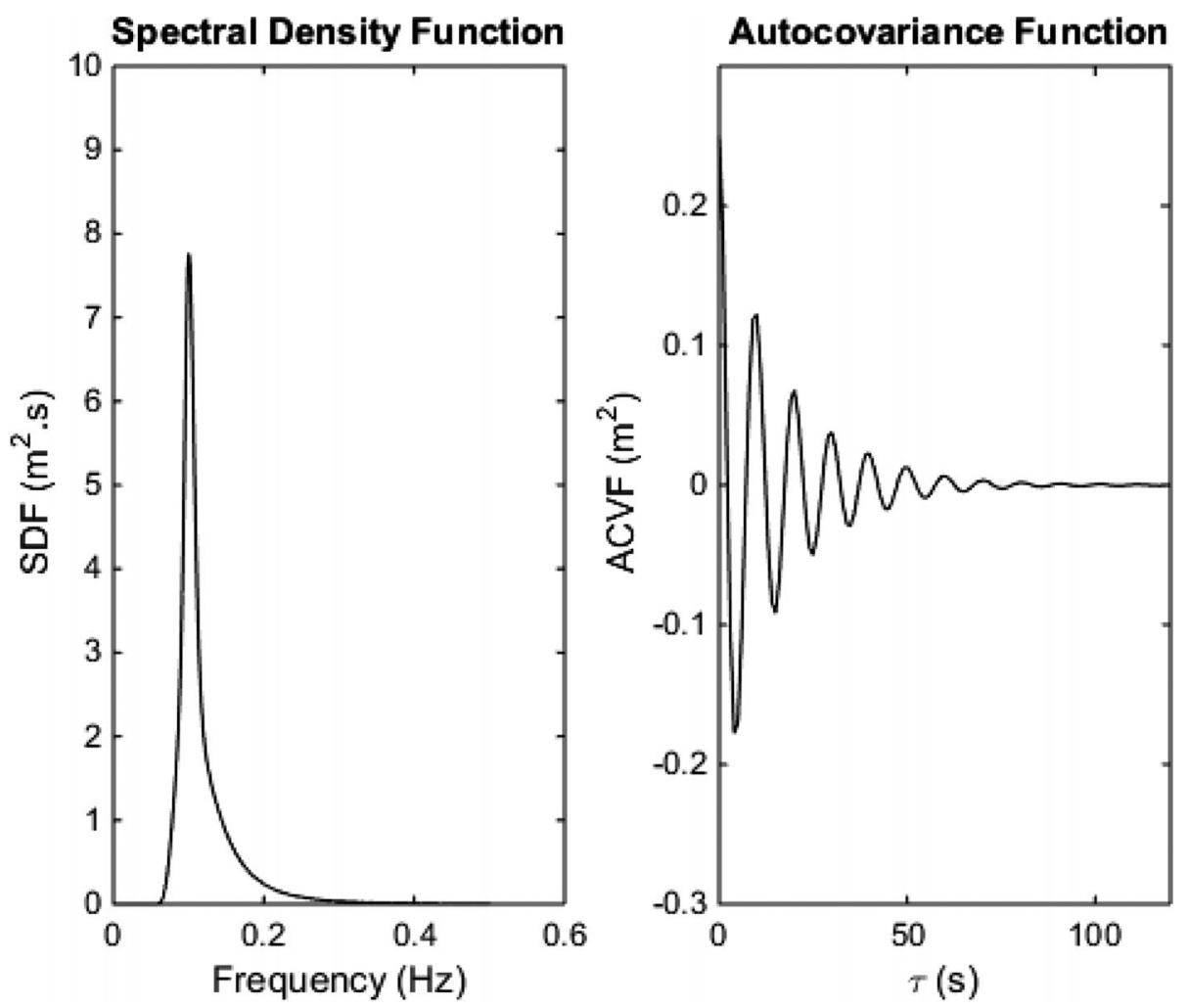

Fig. 1. SDF and ACVF for a JONSWAP spectrum with $H_{s}=2 \mathrm{~m}$ and $T_{p}=10 \mathrm{~s}$.

As an example, a Joint North Sea Wave Project (JONSWAP) SDF [10] with $H_{s}=2 \mathrm{~m}$ and $T_{p}=10 \mathrm{~s}$ is represented in Fig. 1, along with the associated ACVF. Typically, the ACVF of ocean waves fades out to zero after a few dozen seconds.

Clearly, using solely measurement instants, which exhibit no correlation with the prediction time of interest, cannot bring any useful statistical information. For example, from Fig. 1, using only points that are more than $100 \mathrm{~s}$ in the past is not statistically informative to predict current wave elevation values.

However, if such distant points are used, in combination with others that are correlated with the prediction instants, the distant points may still improve the prediction accuracy. In other words, in relation to Fig. 1, measuring wave elevation values beyond $100 \mathrm{~s}$ in the past may be beneficial, if more recent measurements are also taken into account. Thus, the relationship between the shape of the covariance function and the wave signal predictability using a given combination of measurements is not a straightforward problem. A simple look at the covariance function is not sufficient to accurately determine an appropriate set of measurements. Using again the example of Fig. 1, it is yet impossible to ascertain how many points in the past should be used, for the prediction of present and future values, in spite of the ACVF fading out to zero beyond $100 \mathrm{~s}$.

\section{Spatio-Temporal Properties of the Wave Field in Unidirectional Wave Spectra}

The properties of the wave field, both in time and space, are now considered in the simplified case where all waves are assumed to come from the same direction, say the $x$-axis $\vec{u}_{x}$. The wave dispersion equation defines how the frequency $\omega$ and wave number $k$ are related to each other. In the general case, for intermediate water depths, the dispersion relation is written as follows:

$$
\omega^{2}=g k \tanh (h k)
$$

where $g$ is the gravitational constant and $h$ is the water depth. In practice, (4) defines an implicit function $\tilde{k}(\omega)$, which can be numerically evaluated for any arbitrary $\omega$.

In deep water, (4) can be approximated by means of the following explicit relationship:

$$
\tilde{k}(\omega)=\frac{\omega^{2}}{g} .
$$

Given the unidirectionality of the wave field following $\vec{u}_{x}$, the free-surface elevation at a given point only depends on the time considered and point spatial coordinate along the $x$-axis.

The space-time covariance $R_{\eta \eta}$ only depends on the time difference and relative position (projected onto the $x$-axis) because of the stationarity and homogeneity assumptions. Thus, $R_{\eta \eta}$ can be written as a function of $\rho$, which denotes the relative $x$-coordinate, and $\tau$, which denotes the relative measurement time. Using the Wiener-Khintchine relation [8], the covariance between the wave elevation measured at two points, with relative coordinate $\rho$ along the $x$-axis, and at times separated by $\tau$, can be computed as follows from the wave spectrum:

$$
R_{\eta \eta}(\rho, \tau)=\int_{0}^{\infty} S_{\eta \eta}(\omega) \cos (\tilde{k}(\omega) \rho-\omega \tau) d \omega .
$$




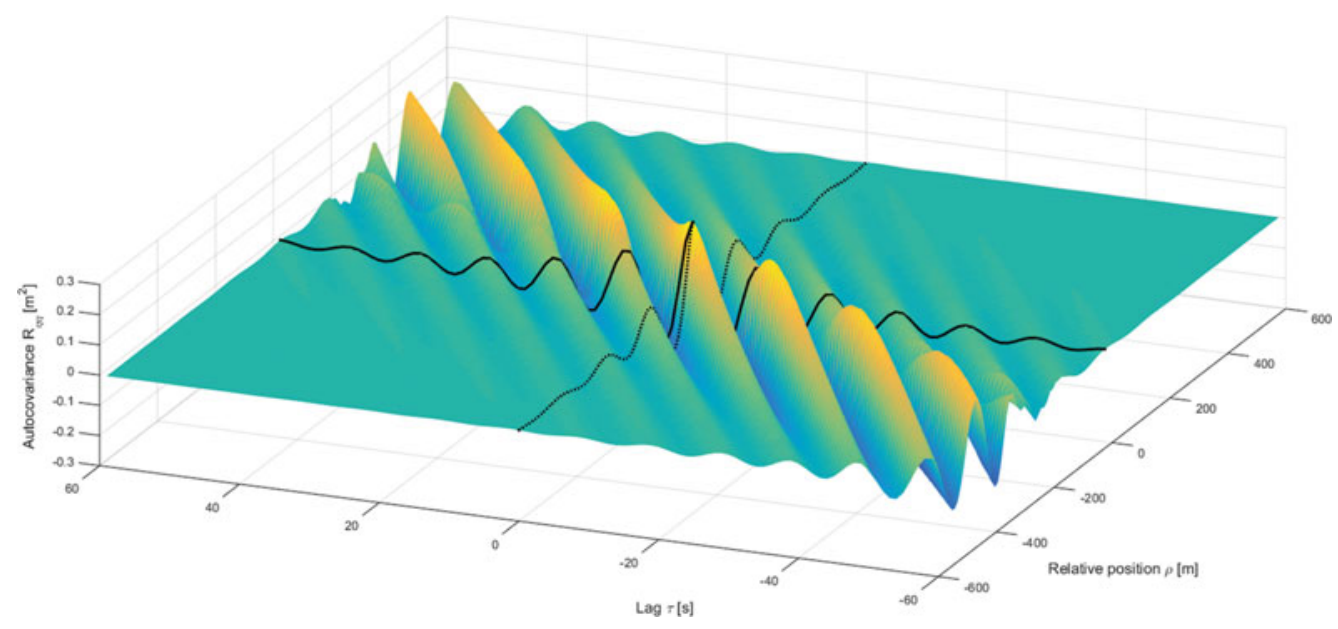

Fig. 2. Space-time ACVF (unidirectional JONSWAP spectrum with $H_{s}=2 \mathrm{~m}$ and $T_{p}=10 \mathrm{~s}$ ).

Using (6), the space-time covariance function of the unidirectional wave field can be computed for any $\rho$ and $\tau$. Fig. 2 shows $R_{\eta \eta}$ for a JONSWAP spectrum [10], with significant wave height $H_{s}=2 \mathrm{~m}$ and peak wave period $T_{p}=10 \mathrm{~s}$, in the water depth $h=200 \mathrm{~m} . R_{\eta \eta}$ reaches its maximum at $(\rho, \tau)=(0,0)$, where its value is $m_{0}=0.25 \mathrm{~m}^{2}$. The usual time-domain ACVF, for point measurements [11], which can be read in the plane defined by $\rho=0$, is highlighted, as well as the space autocovariance, which is shown as a dotted line in the plane defined by $\tau=0$.

Several other comments are appropriate, which are given as follows.

1) The distance between the first two peaks, on the timedomain autocovariance, is close to $T_{p}=10 \mathrm{~s}$.

2) The distance between the first two peaks, on the spatial domain autocovariance, is close to the typical wavelength, i.e., $\lambda_{p}=(g / 2 \pi) T_{p}^{2} \approx 150 \mathrm{~m}$.

3) The main ripples are parallel to a direction with a slope close to the peak phase velocity, for waves with period $T_{p}: c_{\phi} \approx(g / 2 \pi) T_{p} \approx 15 \mathrm{~m} \cdot \mathrm{s}^{-1}$.

4) The strongest peaks and troughs follow a direction with a slope close to half the peak phase velocity, which corresponds to the peak group speed $c_{g}$ in deep water. This is a specific manifestation of the fact that wave energy propagates at the wave group speed. This also suggests that the time-distance predictability domain is governed by the group speed rather than the phase velocity, and thus corroborates the conclusions of other studies, e.g., as in [12]. The relationship between the wave group speed and predictability will be further examined in Section V.

In Fig. 2, the half-plane with $\tau \leq 0$ (resp. $\tau \geq 0$ ) indicates the covariance of the present free-surface elevation, with measurements taken in the past (resp. in the future). The half-plane with $\rho \leq 0$ (resp. $\rho \geq 0$ ) indicates the covariance of the free-surface elevation at the point of interest, with measurements taken upwave (resp. down-wave). It can be seen that the main covariance peaks and troughs are observed for past, up-wave measurements, and future, down-wave measurements (obviously not available in practice).

Therefore, it is statistically informative to use past measurements at points located up-wave, to predict wave elevation values at the location of interest. For example, the wave elevation, at the time and location of interest, presents a strong degree of correlation with the measurement, taken 450 -m up-wave, $60 \mathrm{~s}$ into the past.

In contrast, down-wave measurement points are significantly less informative. In particular, those which are located more than one or two typical wavelengths down-wave do not provide any relevant information for prediction purposes. However, and perhaps not so intuitively, points which are located down-wave, but reasonably close to the point of interest, do bring some exploitable statistical information. ${ }^{2}$

\section{Spatio-Temporal Properties of the Wave Field in Directional Wave Spectra}

Actual ocean waves are reasonably described as a superposition of wave fields coming from multiple directions; therefore, a directional spectrum $S(\omega, \theta)$ can be defined [8], where $\theta$ denotes an angle relative to, say, $\vec{u}_{x}$. Then, the covariance of the wave elevation field, between two points $A$ and $B$ with coordinates $\left(x_{A}, y_{A}, t_{A}\right)$ and $\left(x_{B}, y_{B}, t_{B}\right)$, respectively, depends on the relative coordinates of $A$ and $B$ and time lag $t_{B}-t_{A}$. Denoting $\chi$ and $\psi$ as the relative $x$ and $y$ coordinates, respectively, the space-time covariance function can be derived from the directional spectrum as follows [13]:

$$
\begin{aligned}
R_{\eta \eta}(\chi, \psi, \tau)= & \int_{-\pi}^{\pi} d \theta \int_{0}^{\infty} d \omega S(\omega, \theta) \cos \\
& \times[\tilde{k}(\omega)(\chi \cos \theta+\psi \sin \theta)-\omega \tau] .
\end{aligned}
$$

\footnotetext{
${ }^{2} \mathrm{~A}$ practical consequence is that, considering two measurement points $A$ and $B$, with $A$ up-wave with respect to $B$, then (unsurprisingly) not only the measurements taken at $A$ can contribute to improving wave elevation forecasts at $B$, but also (more surprisingly) the measurements taken at $B$ can also, although to a lesser extent, contribute to improving wave elevation forecasts at $A$.
} 

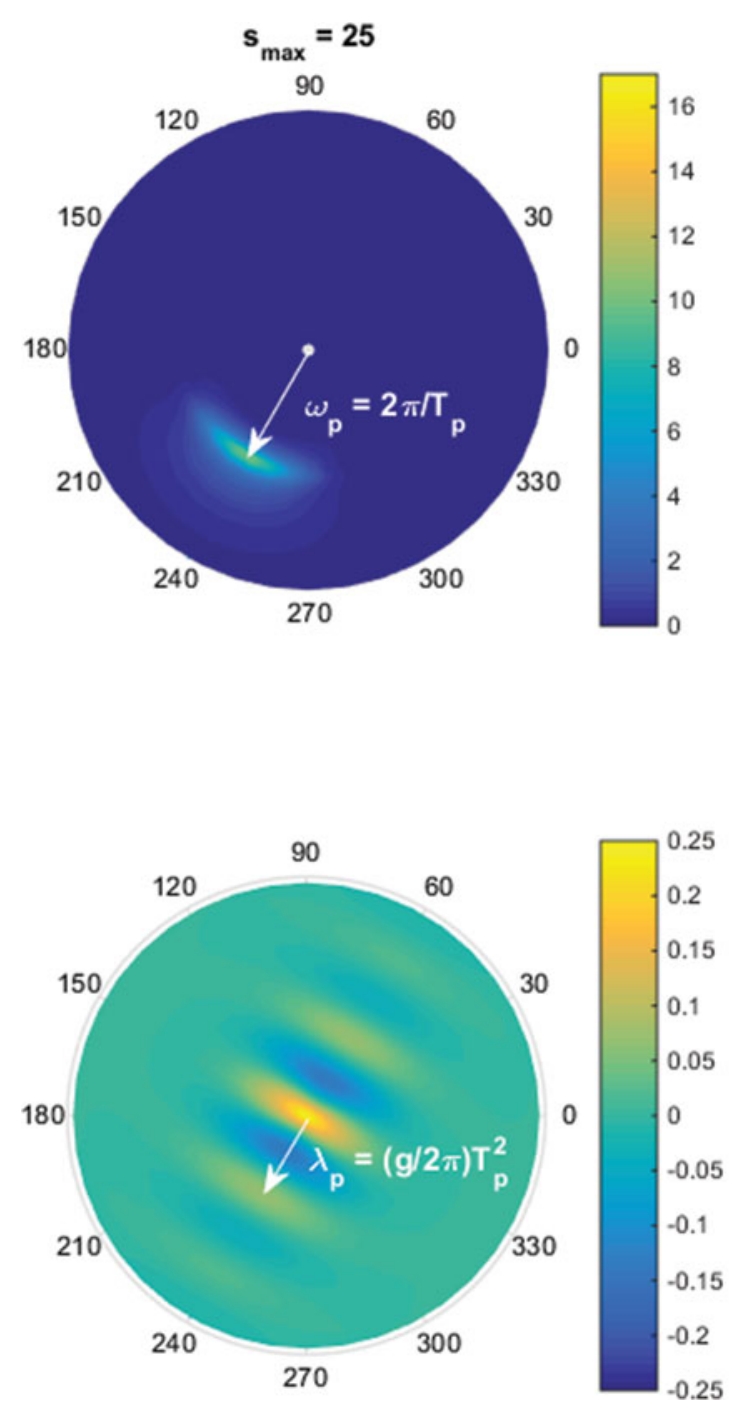
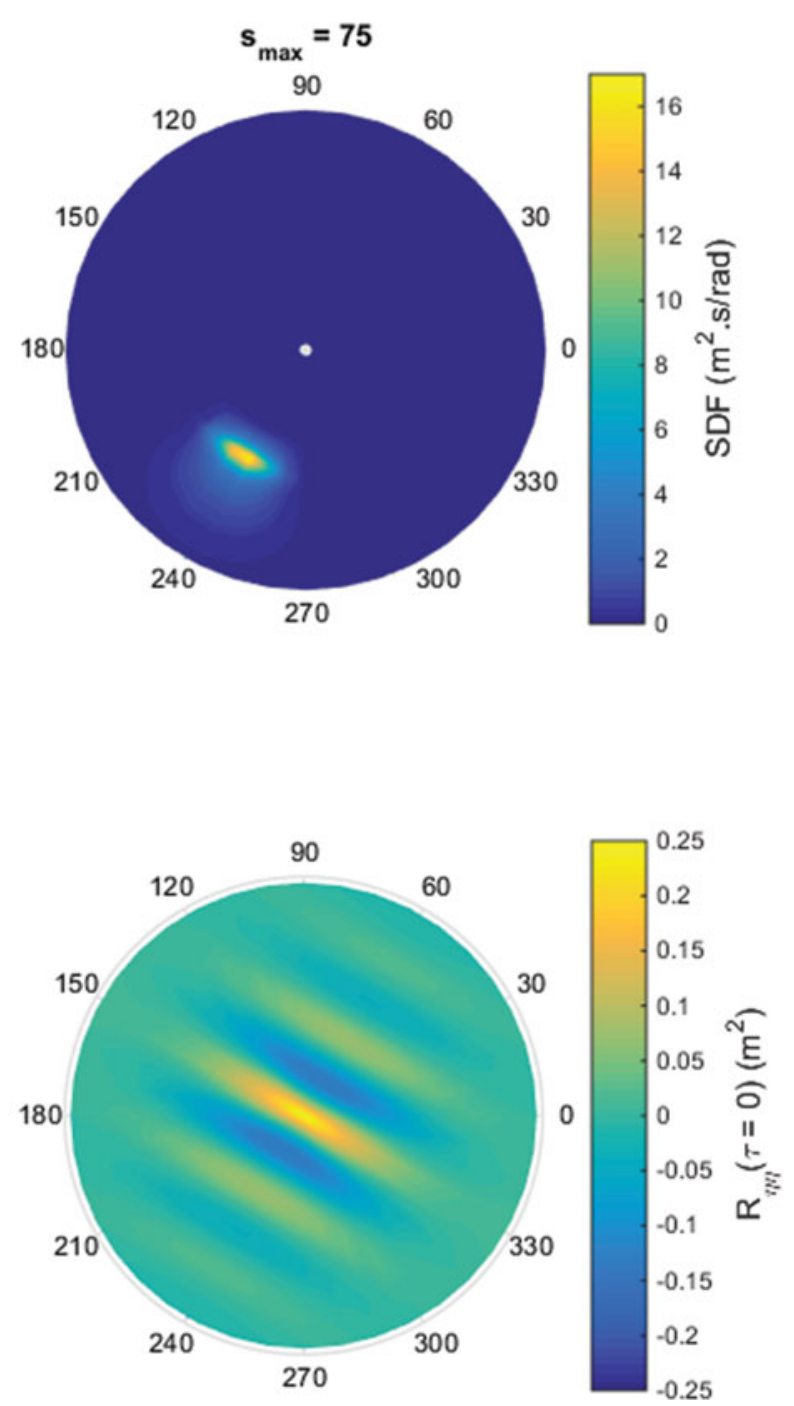

Fig. 3. Examples of the directional JONSWAP spectra with various directional spreading parameters $s$.

The directional spectrum is often described by means of a parametric, directional spreading function $D(\omega, \theta)$ such that

$$
S(\omega, \theta)=D(\omega, \theta) S(\omega)
$$

where $S(\omega)$ is the omnidirectional spectrum and, $\forall \omega$

$$
\int_{-\pi}^{\pi} D(\omega, \theta) d \theta=1
$$

Several formulations exist for the spreading function $D(\omega, \theta)$, such as the cosine-square, Mitsuyasu, Hasselmann, and Borgman's formulas [8].

In the numerical applications of this paper, the Mitsuyasu formula is retained since it is widely used for engineering applications and is more realistic than the cosine-square formulation [14]. Note, however, that the use of the Mitsuyasu formula and, more generally, the assumption of a directional spectrum in the form of (8) are not necessary for the application of (7), and hence for the derivation of the theoretical results presented in this paper. The Mitsuyasu formulation is rather chosen as a simple and an easily tuneable example.
Denoting $\theta_{p}$ as the peak wave direction and $\omega_{p}=2 \pi / T_{p}$, the Mitsuyasu directional spreading function is given as follows:

$$
D(\theta, \omega)=\frac{2^{2 s-1}}{\pi} \frac{\Gamma(s+1)^{2}}{\Gamma(2 s+1)}\left|\cos \left(\frac{\theta-\theta_{p}}{2}\right)\right|^{2 s}
$$

where the parameter $s$ is frequency-dependent as

$$
s= \begin{cases}s_{\max }\left(\omega / \omega_{p}\right)^{5}, & \text { for } \omega \leq \omega_{p} \\ s_{\max }\left(\omega / \omega_{p}\right)^{-2.5}, & \text { for } \omega>\omega_{p} .\end{cases}
$$

$s_{\max }$ depends on the nature of the wave system considered. A large $s_{\max }$ indicates that a spectrum sharply concentrated around its mean direction. Values such as 10, 25, and 75 for wind waves, swell with short decay distance, and swell with long decay distance, respectively, [14] may be used.

Figure 3 shows two examples of the directional spectra, obtained from the JONSWAP spectrum of Section II-B, with a Mitsuyasu spreading function with $s_{\max }=25$ and $s_{\max }=75$. The corresponding spatial covariance functions $R_{\eta \eta}(\chi, \psi, 0)$ are shown below each spectrum. 

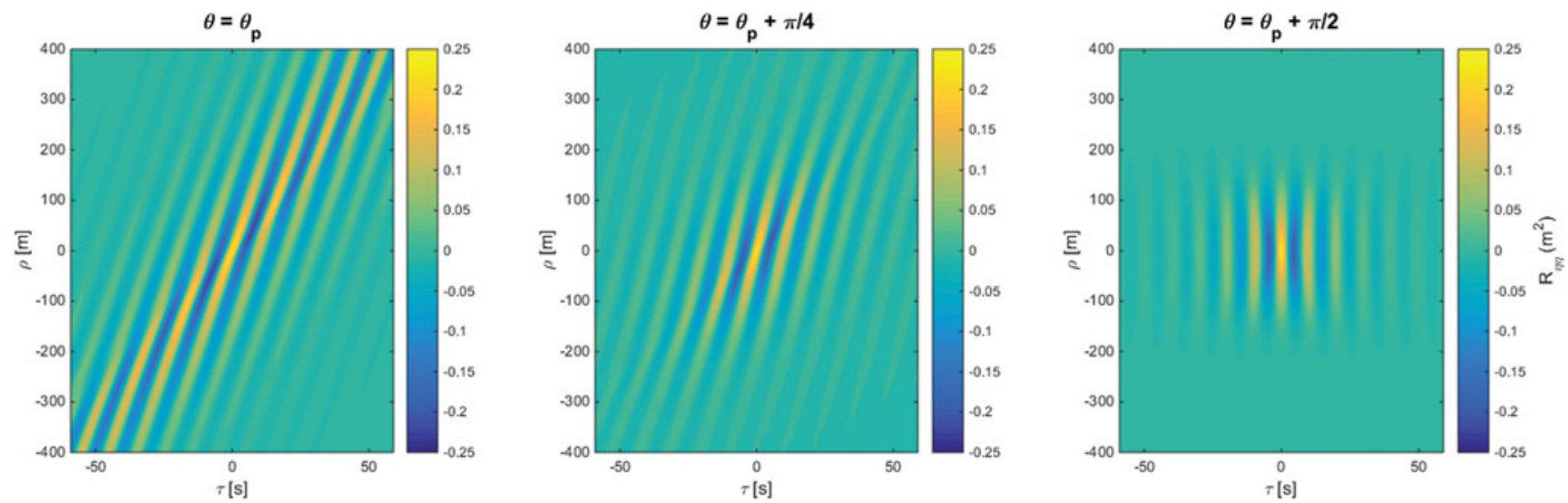

Fig. 4. Space-time covariance for different orientations of the two measurement points (directional JONSWAP spectrum with Mitsuyasu spreading function and parameter $s_{\max }=25$ ).

Similar to Section II-C, the space-time covariance properties of the wave field may be analyzed. To this end, it is interesting to consider two points forming an angle $\theta$ relative to $\vec{u}_{x}$. Then, the space-time covariance can be considered along the direction formed by the two points, and depends on the time lag $\tau$ and relative position $\rho$ along the axis of angle $\theta$. Fig. 4 shows the time-distance covariance function for the directional spectrum of Fig. 3, with spreading parameter $s_{\max }=25$, for two measurement points forming angles of $0^{\circ}, 45^{\circ}$, and $90^{\circ}$, respectively, with mean wave direction $\theta_{p}$. Unsurprisingly, it is in the mean wave direction that the strongest covariance peaks and troughs are found, which means that, for prediction purposes, a measurement point located up-wave, in the mean wave direction, is more informative than a measurement point forming some nonzero angle with respect to $\theta_{p}$.

\section{OPtIMAL WAVE ELEVATION PREDICTOR}

\section{A. Treating Measurements As a Gaussian Vector}

If the wave description, as a stationary, homogeneous, Gaussian random field, is valid (which is the crucial assumption underlying this paper), then following the definition of a Gaussian random field, any finite, discrete combination of measurements, taken at various points in time and space, form a multivariate, Gaussian random vector.

Consider a discrete set of points in time and space. Some of those points, indexed by $m \in \llbracket 1 ; M \rrbracket$, can be observed (because they are "in the past" and at a location where the free-surface elevation can be measured) and are termed measurement points, whereas some other points, indexed by $n \in \llbracket 1 ; N \rrbracket$, have to be predicted (prediction points) because they are "in the future" and/or at a location where the free-surface elevation cannot be observed. The $M$ measurement points form a vector $\mathbf{x} \in \mathbb{R}^{M}$ and the $N$ prediction points form a vector $\mathbf{y} \in \mathbb{R}^{N}$.

Altogether, the $N+M$ points form a multivariate Gaussian random vector $\mathbf{v} \in \mathbb{R}^{M+N}$. Its mean is $0_{\mathbb{R}^{M+N}}$ and its variancecovariance matrix, noted $\Sigma_{\mathrm{vv}}$, can be entirely derived from the wave spectrum, i.e., from the wave covariance values between any pair of points in time and space, which depends on the relative position and time of any pair of points, as in (6) and (7).

$\Sigma_{\mathbf{v v}}$ can be written as follows:

$$
\Sigma_{\mathrm{vv}}=\left(\begin{array}{cc}
\Sigma_{\mathrm{yy}} & \Sigma_{\mathrm{yx}} \\
\Sigma_{\mathrm{xy}} & \Sigma_{\mathrm{xx}}
\end{array}\right)
$$

where $\Sigma_{\mathbf{y x}}=\Sigma_{\mathbf{x y}}^{\mathrm{T}}$.

Using $\mu_{\mathbf{x}}=\mu_{\mathbf{y}}=0$, the conditional distribution of $\mathbf{y} \mid \mathbf{x}$ is a multivariate Gaussian (see, for example, [15]) with mean

$$
\mu_{\mathbf{y} \mid \mathbf{x}}=\Sigma_{\mathbf{y x}} \Sigma_{\mathbf{x x}}^{-1} \mathbf{x}
$$

and variance (assuming $\Sigma_{\mathrm{xx}}$ is nonsingular)

$$
\Sigma_{\mathbf{y} \mid \mathbf{x}}=\Sigma_{\mathbf{y y}}-\Sigma_{\mathbf{y x}} \Sigma_{\mathbf{x x}}^{-1} \Sigma_{\mathbf{x y}} .
$$

Therefore, the best predictor of $\mathbf{y}$, in a least mean square sense, is given as

$$
\hat{\mathbf{y}}=\mu_{\mathbf{y} \mid \mathbf{x}}=\Sigma_{\mathbf{y x}} \Sigma_{\mathbf{x x}}^{-1} \mathbf{x} .
$$

The mean square prediction error, for each prediction point in time and space, is given by the diagonal terms of $\Sigma_{\mathbf{y} \mid \mathbf{x}}$. For a given choice of $M$ measurement points in time and space, any other forecasting method is suboptimal with respect to the law derived in (15), to evaluate the $N$ prediction points.

It is interesting to examine the behavior of the optimal predictor, when the prediction points are at large distances or time lags from the measurement points. In such cases, the rows of $\Sigma_{\mathrm{yx}}$, which contain covariance values between measured and predicted points, take values close to zero. Therefore, from (15), the optimal predictor fades out to zero for predicted points located at large distances from the measured points, or far ahead in time. This is statistically consistent since, the wave field being zero mean, the best possible guess, for free-surface elevation values at large time horizons or large distances from the observed points, is always zero. Other prediction methods do not necessarily share this desirable property: for example, AR model predictions, propagated ahead in time, do not generally tend to zero; it is not the case either for physical wave propagation models since the sinusoidal terms do not fade out to zero for large distances or time horizons. 


\section{B. Discarding Statistically Redundant Information}

Equation (15) holds, provided that $\Sigma_{\mathrm{xx}}$ is invertible. However, it may happen that $\Sigma_{\mathrm{xx}}$ is singular or badly conditioned, which means that the components of $\mathrm{x}$ are not statistically independent. To discard the statistically redundant information contained in $\mathbf{x}$, the measured values can be transformed in a manner described as follows.

$\Sigma_{\mathrm{xx}}$ is a variance-covariance matrix; it is therefore symmetric, positive semidefinite and can be diagonalized: $\Sigma_{\mathrm{xx}}=$ $Q \Lambda Q^{\mathrm{T}}$, where $\Lambda$ is the diagonal matrix of positive eigenvalues $\left\{\lambda_{1}, \ldots, \lambda_{M}\right\}$ arranged in descending order, $Q^{\mathrm{T}}=Q^{-1}$, and the $i$ th column of $Q$ is the eigenvector $\mathbf{q}_{i}$.

Next, only $M^{\prime}$ nonzero eigenvalues ${ }^{3}$ are selected. Define $\Lambda^{*}$, the diagonal matrix of the retained eigenvalues, and $Q^{*} \in$ $\mathbb{R}^{M \times M^{\prime}}$, composed of the eigenvectors $\mathbf{q}_{i \in \llbracket 1 ; M^{\prime} \rrbracket}$ corresponding to $\Lambda^{*}$. Define

$$
A=\left(\begin{array}{cc}
\mathbb{I}_{\mathbb{R}^{N \times N}} & 0_{\mathbb{R}^{N \times M}} \\
0_{\mathbb{R}^{M^{\prime} \times N}} & Q^{* T}
\end{array}\right) .
$$

Define $\mathbf{v}^{\prime}:=A \mathbf{v}$ and $\mathbf{x}^{\prime}:=Q^{* T} \mathbf{x} . \mathbf{v}^{\prime}$ is a Gaussian random vector, and its variance-covariance matrix has the following structure:

$$
\Sigma_{\mathbf{v}^{\prime} \mathbf{v}^{\prime}}=A \Sigma_{\mathbf{v} \mathbf{v}} A^{T}=\left(\begin{array}{cc}
\Sigma_{\mathbf{y y}} & \Sigma_{\mathbf{y x}^{\prime}} \\
\Sigma_{\mathbf{x}^{\prime} \mathbf{y}} & \Lambda^{*}
\end{array}\right)
$$

where

$$
\Sigma_{\mathbf{y x}^{\prime}}=\Sigma_{\mathbf{y x}} Q^{*}=\Sigma_{\mathbf{x}^{\prime} \mathbf{y}}^{\mathrm{T}} .
$$

Then, (14) and (15) can be safely applied to the new vector $\mathbf{v}^{\prime}$. The best predictor is now given as

$$
\hat{\mathbf{y}}=\Sigma_{\mathbf{y x}^{\prime}} \Sigma_{\mathbf{x}^{\prime} \mathbf{x}^{\prime}}^{-1} \mathbf{x}^{\prime}=\Sigma_{\mathbf{y x}} Q^{*} \Lambda^{*(-1)} Q^{* T} \mathbf{x}
$$

without any loss of accuracy since only statistically redundant information has been eliminated in $\mathbf{x}$.

In the following, the matrix $P=\Sigma_{\mathbf{y x}} Q^{*} \Lambda^{*(-1)} Q^{* T}$ will be termed the prediction matrix as it is the matrix that linearly transforms a set of measurements into a set of predictions.

\section{Performance of the Optimal Predictor For Point MEASUREMENTS}

In this section, the "looking backward" approach is considered from the spectrum-based optimal predictor perspective, where the spatial location of measurement and prediction points is identical. Measurement points simply consist of the past $M$ wave elevation values at the location of interest, and prediction points are the next $N$ wave elevation values.

The JONSWAP spectrum, shown in Fig. 1, is used as an example. For different orders $M$, the optimal predictor is derived as in (15), and the associated error $e^{2}(h)=\mathbb{E}[(\hat{\eta}(n+h)-\eta(n+$ $h))^{2}$ ] is calculated for each time horizon between 0 and $60 \mathrm{~s}$, without requiring any simulation since the sequence $e^{2}(h)$ is readily provided by the diagonal terms of $\Sigma_{\mathbf{y} \mid \mathbf{x}}$ in (14). The

\footnotetext{
${ }^{3}$ Or eigenvalues larger than some small threshold.
}

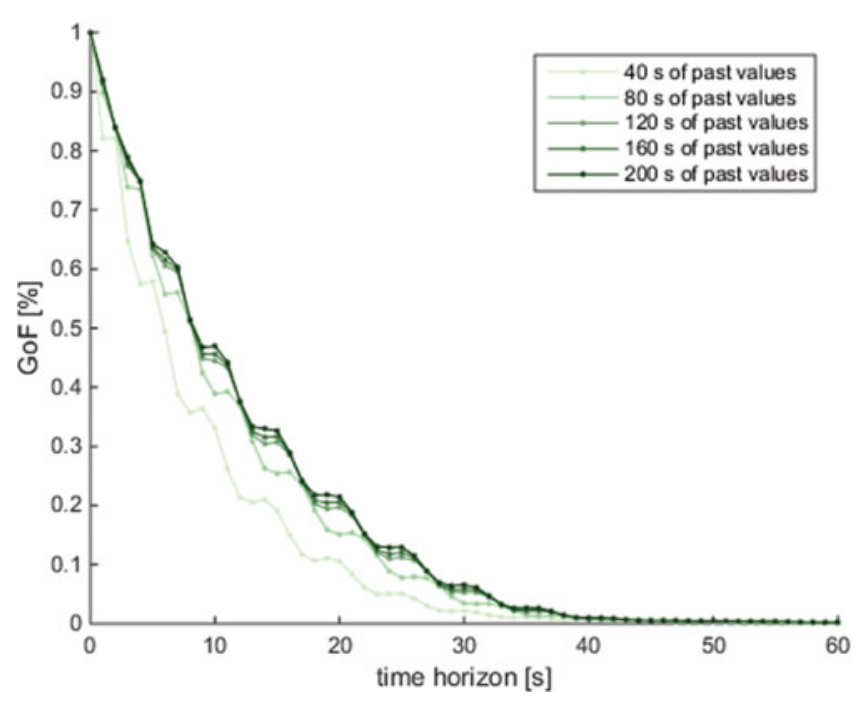

Fig. 5. GoF of the optimal predictor for different time horizons and predictor orders (JONSWAP spectrum with $H_{s}=2 \mathrm{~m}$ and $T_{p}=10 \mathrm{~s}$ ).

goodness of fit (GoF) of the prediction defined as

$$
G(h)=1-\sqrt{\frac{e^{2}(h)}{\mathbb{E}\left[\eta^{2}\right]}}
$$

is plotted in Fig. 5 for different orders $M$.

It can be seen in Fig. 5 that the GoF improves with the predictor order. However, beyond some value of $M$, the improvement achieved by increasing the order is not significant. Considering the specific example of Fig. 5 using data for more than $120 \mathrm{~s}$ in the past does not significantly improve the forecast accuracy.

The quality of the forecast decreases rapidly with the time horizon considered. For a time horizon of $10 \mathrm{~s}$, which corresponds to one typical wave period for the spectrum considered, the GoF is less than $50 \%$, which means that the RMS error committed is half the variance of the signal itself.

JONSWAP spectra are idealized spectra, with energy sharply concentrated at low frequencies, which is beneficial in terms of wave forecasts. It can be expected that with actual wave spectra, which also include significant higher frequency content, the optimal predictor would exhibit a smaller GoF. This is illustrated in Fig. 6 where, using $120 \mathrm{~s}$ of past data, the GoF of the optimal predictor is shown for two different spectra: the JONSWAP spectrum of Fig. 1 and the same spectrum where higher frequency components have been added. It can be seen that the quality of the forecast is significantly affected by the presence of high-frequency components.

In fact, the measured input may not be the wave elevation itself but, say, the linear wave excitation force [5] acting on a WEC. The SDF of such an input depends on the wave elevation SDF, transformed depending on the mechanical properties of the WEC system. Typically, high-frequency components may be filtered out by the WEC natural dynamics, and therefore the excitation force may have a better predictability than the wave elevation itself. 

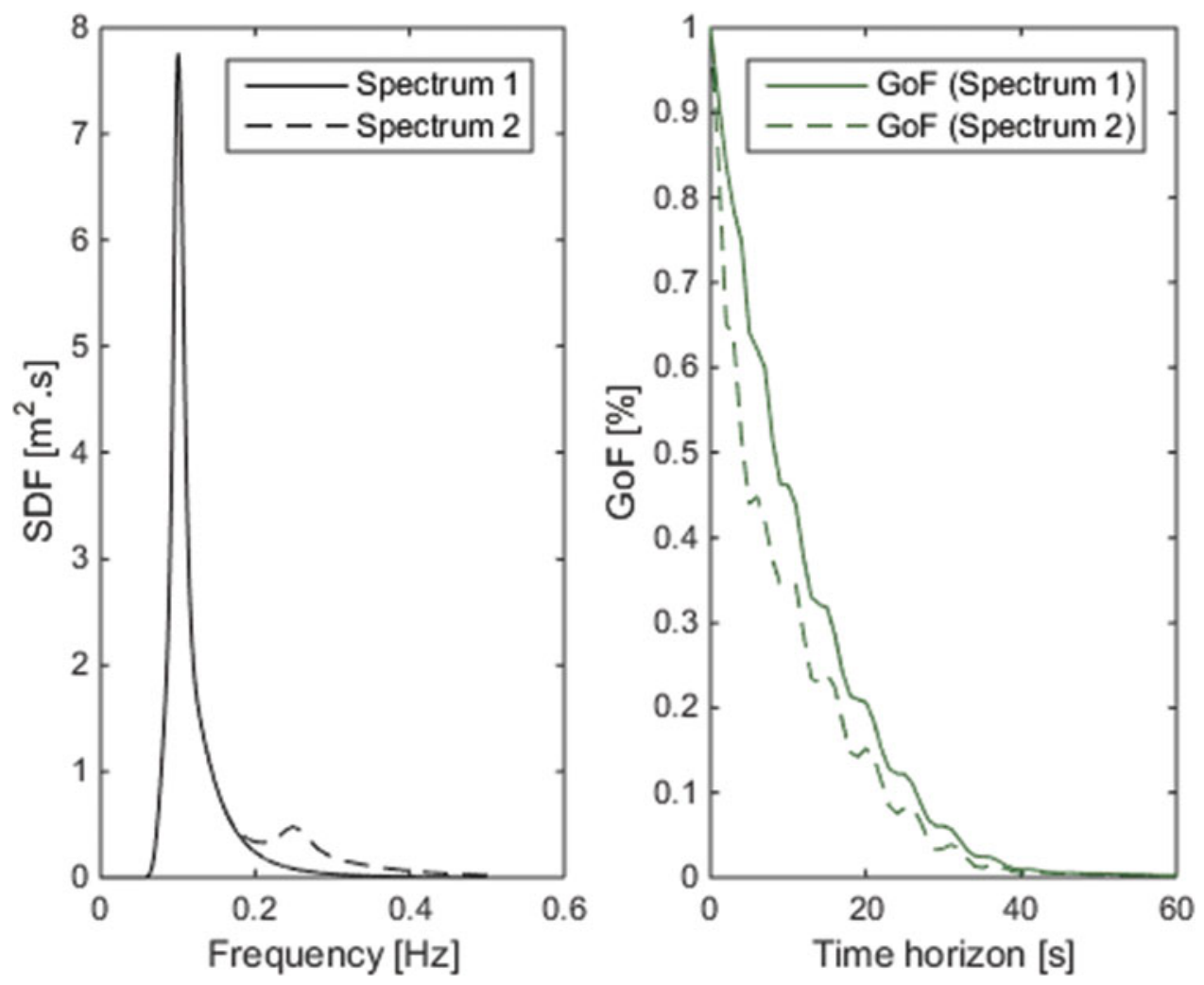

Fig. 6. GoF of the optimal predictor, using $120 \mathrm{~s}$ of past values, for two different spectra.

Considering Fig. 5, it is unlikely that accurate wave elevation forecasts can be achieved for prediction horizons of more than a typical wave period, which is clearly not compatible with applications such as quiescent period detection for maritime operations (which require reasonably accurate forecasts more than $1 \mathrm{~min}$ ahead). The compatibility of such short time horizons with real-time WEC control is also questionable since it is suggested in [6] that wave elevation values up to three typical wave periods into the future may be necessary for the controller to perform optimally.

\section{Performance of the Optimal Predictor in UNIDIRECTIONAL WAVE SPECTRA}

In this section, the JONSWAP unidirectional wave spectrum studied in Section II-C is considered. The wave elevation is predicted at one specific point $A$, for various time horizons, using wave elevation values measured at a point $B$ located at various distances, up-wave or down-wave, with respect to $A$. Thus, applying the terminology used in Section III, the vector $\mathrm{y}$ consists of the wave elevation at $A$, for various time horizons, and the vector $\mathbf{x}$ is composed of past measurements at location $B$.

Fig. 7 shows the GoF of the prediction obtained in $A$, for various locations of $B$ up-wave and down-wave, taking as input (in the vector $\mathbf{x}$ ) $300 \mathrm{~s}$ of past values measured at $B$. It appears that the forecast time horizon can be arbitrarily increased by measuring the waves at longer distances up-wave of $A$. Since the full signal energy travels between $B$ and $A$, measuring the

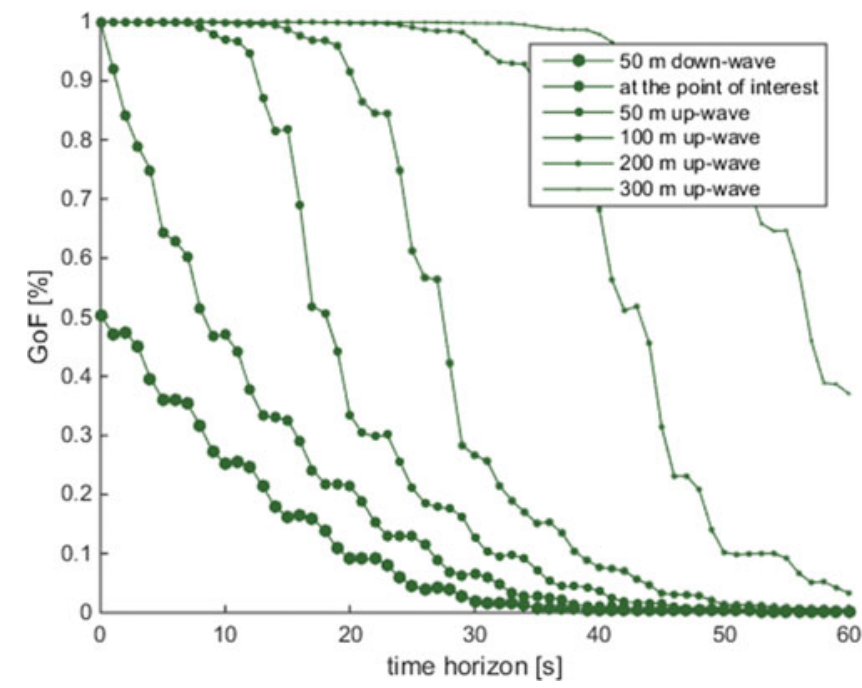

Fig. 7. GoF of the prediction at a point $A$ using a measurement point $B$ at various relative positions along the wave direction (unidirectional JONSWAP, $\left.H_{s}=2 \mathrm{~m}, T_{p}=10 \mathrm{~s}\right)$.

signal over a long enough period even allows for a deterministic prediction over some duration. Unsurprisingly, down-wave measurements are of little help in forecasting the wave elevation at $A$; however, some usable statistical information is present in the down-wave signal, as evidenced by the nonzero value of the GoF for short time horizons, thus confirming what could be expected from the analysis of Fig. 2. 

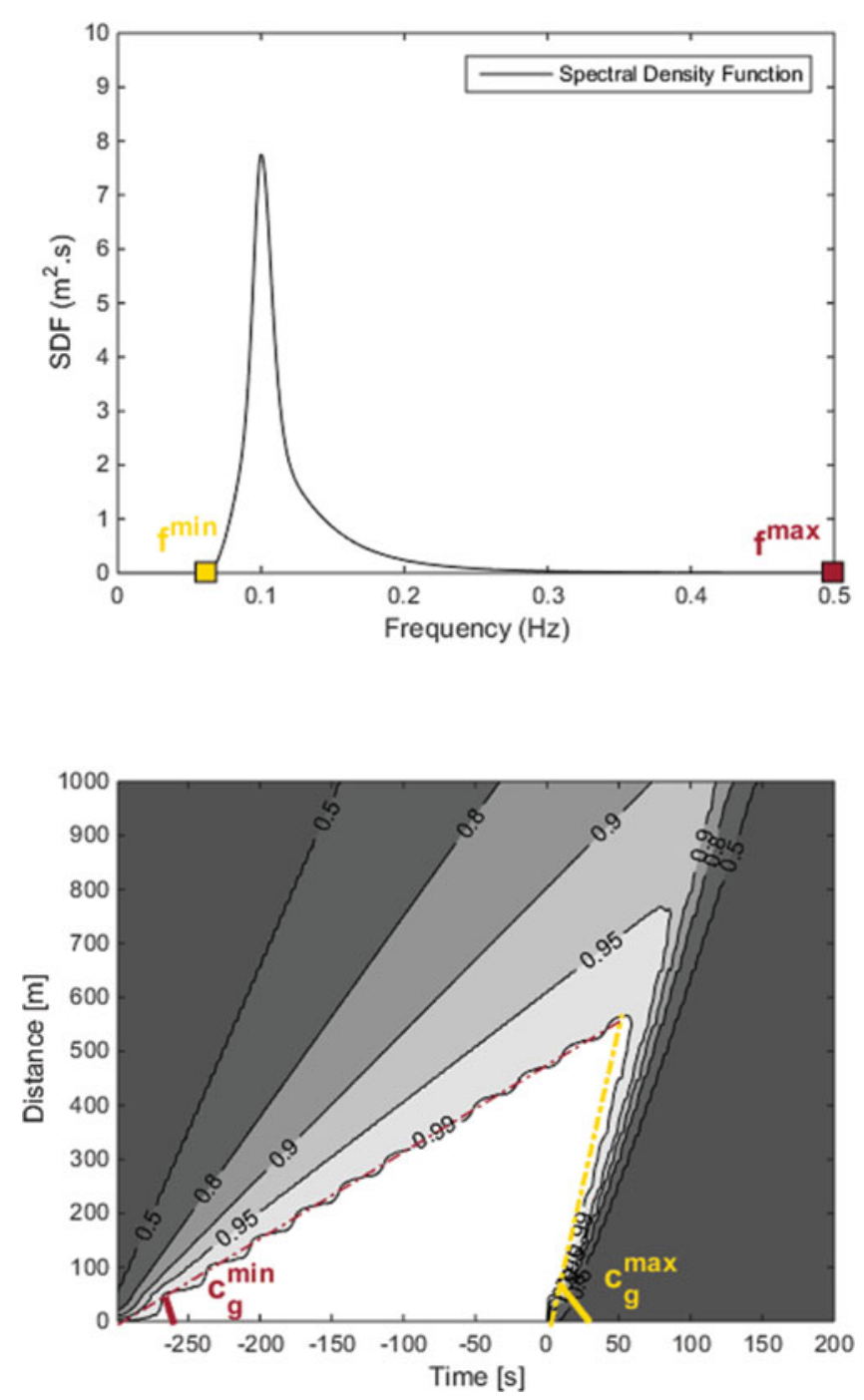

Fig. 8. GoF of the prediction, using up-wave measurements, for various time horizons and relative positions (unidirectional JONSWAP, $H_{s}=2 \mathrm{~m}, T_{p}=$ $10 \mathrm{~s})$.

A slightly different perspective may be adopted by fixing the measurement location $B$ and the duration over which past values are recorded and computing the GoF of the prediction for various time horizons and down-wave locations. This is shown in Fig. 8 for $300 \mathrm{~s}$ of measured values. A distinctive timedistance "predictable zone" appears, where the wave prediction error is approximately zero.

Early research on DSWP, as recalled in [1], indicated some uncertainty as to whether the phase or group speed determines the predictable zone. Using various prediction methods [1], [12], it was proven, both through theoretical considerations and numerical simulations, that the predictable zone is rather governed by the wave group speeds. This latter result is clearly corroborated by the joint examination, in Fig. 8, of the predictable zone obtained with the optimal predictor and the frequency content of the wave spectrum considered. Using the deep-water dispersion relation, the lowest and highest group speeds (corresponding to the highest and lowest frequencies, respectively) are computed as

$$
\left\{\begin{array}{l}
c_{g}^{\max } \approx g /(4 \pi \times 0.06) \approx 13 \mathrm{~m} / \mathrm{s} \\
c_{g}^{\min } \approx g /(4 \pi \times 0.5) \approx 1.5 \mathrm{~m} / \mathrm{s}
\end{array}\right.
$$

and are pictured on the contour plot in Fig. 8 by means of dashed lines with slopes $c_{g}^{\min }$ and $c_{g}^{\max }$.

\section{PERFORMANCE OF THE OPTIMAL PREDICTOR IN DiRECTIONAL WAVE SPECTRA}

In the idealized case of a unidirectional wave field as in Section V, accurate forecasts can be achieved by using measurements at only one up-wave location. In contrast, in a multidirectional wave field, energy travels in a multitude of directions, and therefore, using measurement points along only one orientation may not be sufficient to capture enough statistical information. The effect of spectrum directionality is illustrated in Fig. 9, where waves at the prediction location are forecast using $300 \mathrm{~s}$ of past measurements, taken at a point located at different distances $(100,200$, and $300 \mathrm{~m}$ ) from the prediction point, and forming angles of $0^{\circ}, 45^{\circ}$, and $90^{\circ}$ with respect to the mean wave direction.

As can be observed in Fig. 9, the distance between measurement and prediction points has an adverse effect on the forecast quality. For example, when the measurement point is located as little as $100 \mathrm{~m}$ behind the prediction point, in the mean wave direction, the achievable GoF is barely higher than 50\%. Similar to the unidirectional case, a "plateau" can be identified, whose length increases with the distance to the prediction point. But, in contrast to the unidirectional case, the height of the plateau is less than $100 \%$ and is negatively affected by the distance. The effect of distance is a significant difference with respect to a unidirectional wave model, whereby a deterministic forecast can be achieved for any time horizon, by arbitrarily increasing the distance to the up-wave measurement location.

When the direction formed by the measurement and prediction points departs from the mean wave direction, the predictor performance drops, as might be expected by observing Fig. 4, which shows that measurements, taken off the mean wave direction, provide less statistical information.

Given the poor quality of the achievable forecast using only one measurement location, an array of measurement locations is considered and illustrated in Fig. 10; the points are located at regularly spaced angular positions (every $5^{\circ}$ ) along a semicircle with the prediction point as its center facing the mean wave direction.

Different values for the radius of the semicircle are investigated, and the resulting GoFs are shown in Fig. 11. The GoF is significantly improved with respect to the results obtained using a single measurement location (see Fig. 9). However, the distance has, again, an adverse effect on the height of the plateau.

The effect of the spreading parameter $s_{\max }$ is investigated in Fig. 12. Unsurprisingly, spectra that are sharply concentrated around their mean direction (larger $s_{\max }$ ) offer a more favorable situation than those that have a broader directional distribution (smaller $s_{\max }$ ). 

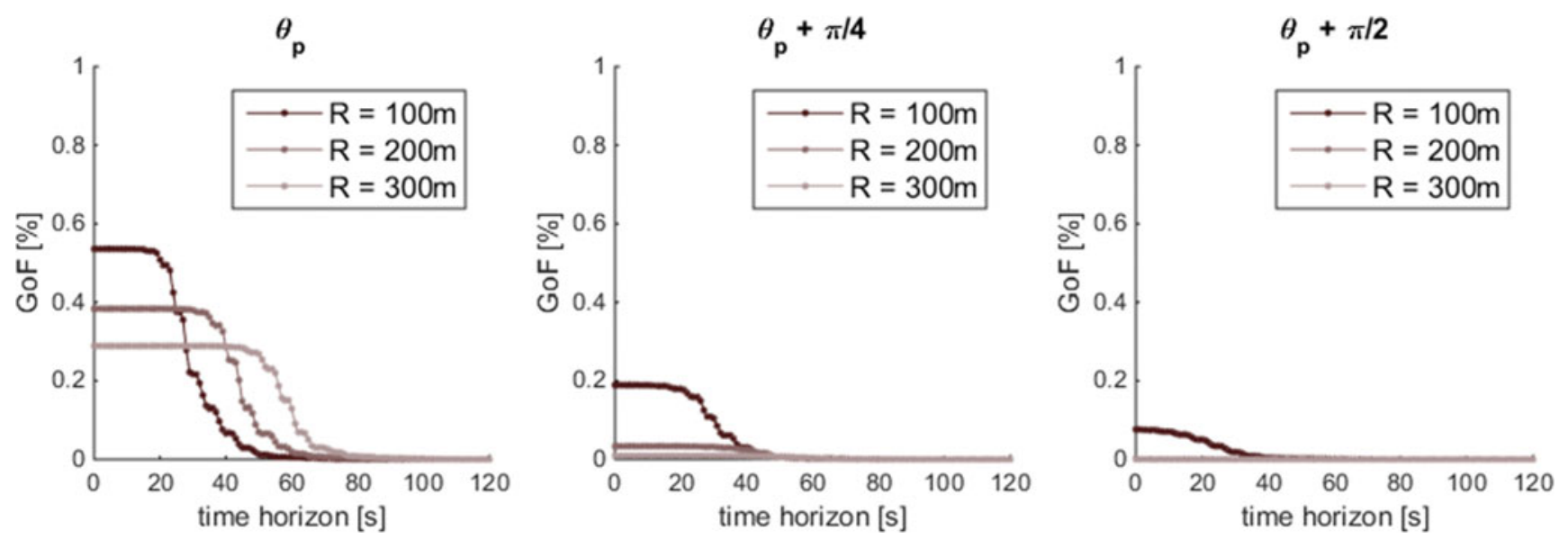

Fig. 9. GoF of the prediction using the one up-wave measurement points located at various distances from the prediction point and at various angles $\left(0^{\circ}, 45^{\circ}\right.$, and $90^{\circ}$ ) with respect to the mean wave direction (directional JONSWAP spectrum, using a Mitsuyasu spreading function with $s_{\max }=25$ ). $300 \mathrm{~s}$ of past measurements are used by the predictor.

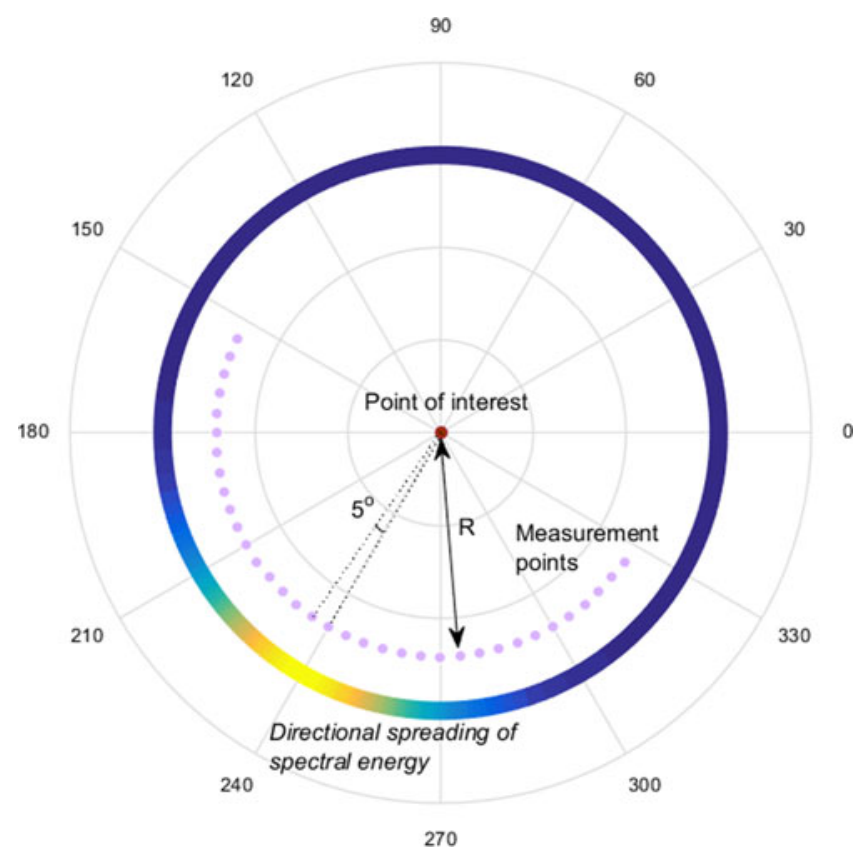

Fig. 10. "Simple" angular layout.

It is possible to improve the measurement array configuration with respect to the simple layout of Fig. 10, so as to capture more information in the directions where the spectrum is concentrated. An improved measurement layout is proposed in Fig. 13, where the angular spacing between two neighboring measurement locations is made smaller close to the mean wave direction. For comparability, the total number of measurement locations in Fig. 13 is kept identical to the one shown in Fig. 10.

The improvement achieved by the new angular layout is shown in Fig. 14. For the spectrum considered, and with a $600-\mathrm{m}$ radius, a GoF close to $80 \%$ seems to be achievable, up to time horizons of the order of $80 \mathrm{~s}$.

Finally, a significantly larger array of measurement points is considered, as shown in Fig. 15, where measurements are taken

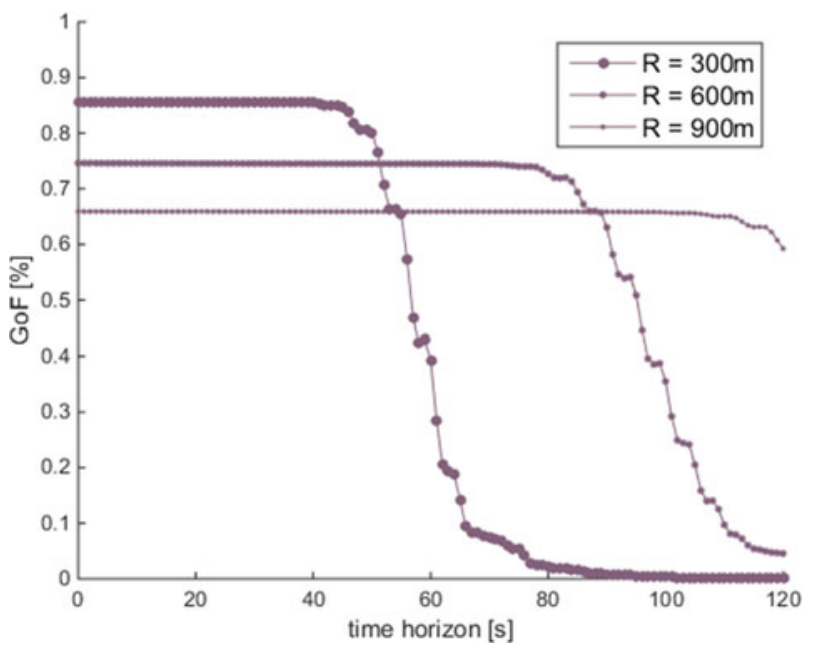

Fig. 11. Effect, on the predictor GoF, of the distance between the point of interest and the measurement points $\left(s_{\max }=25\right.$, "simple" layout).

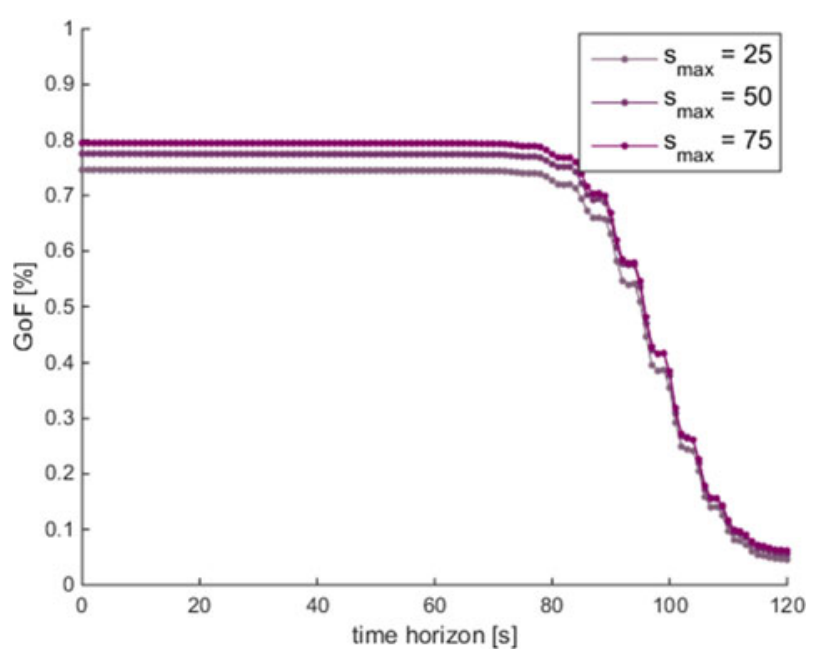

Fig. 12. Effect of the angular spreading on the predictor $\operatorname{GoF}(R=600 \mathrm{~m}$, "simple" layout) - Higher $s_{\max }$ values correspond to more concentrated spectra. 


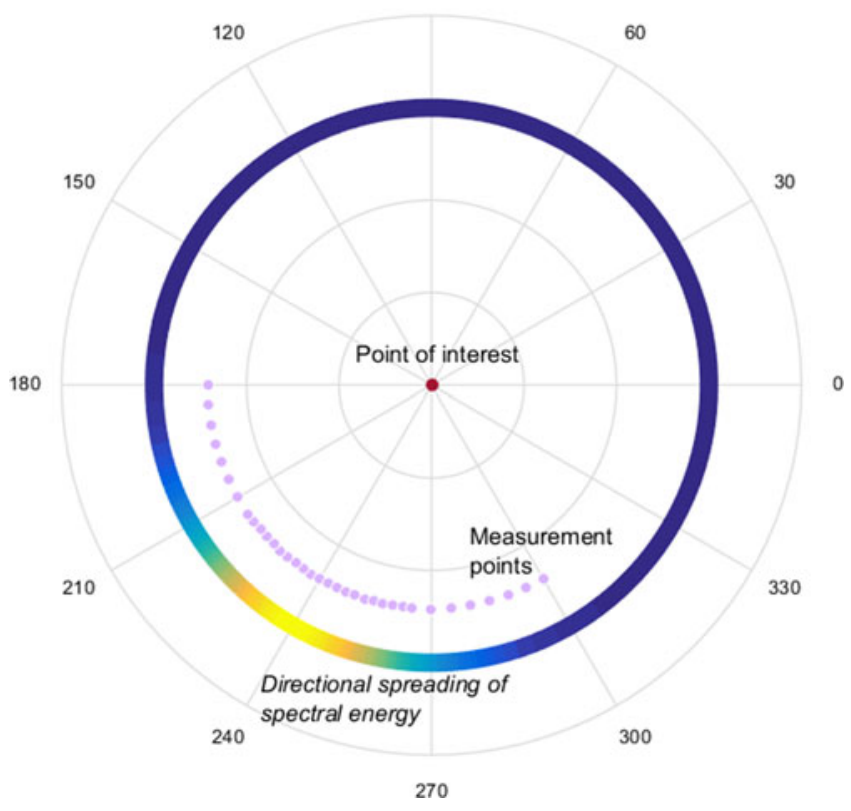

Fig. 13. Improved angular layout (with the same number of points as in the "simple" layout).

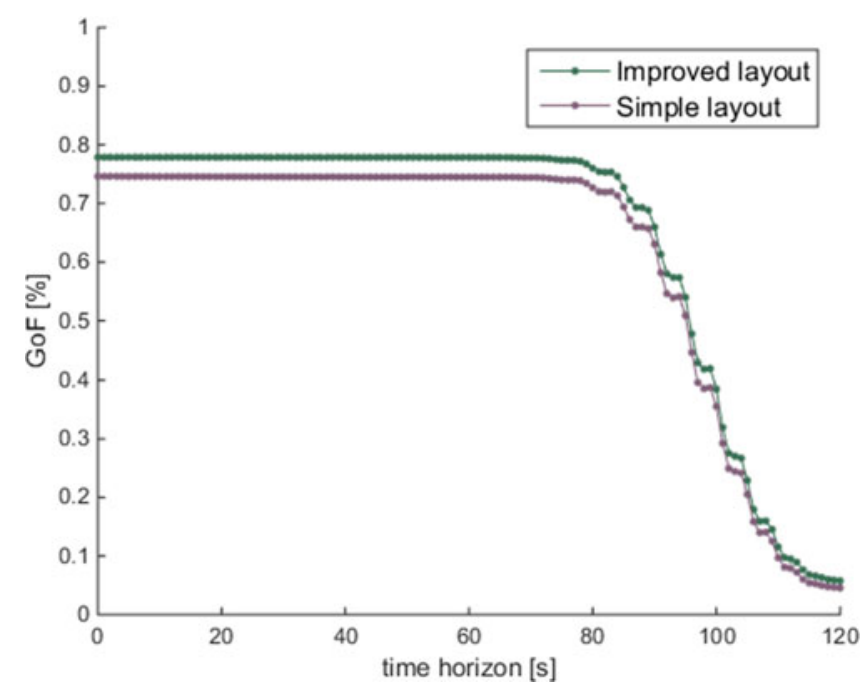

Fig. 14. Effect of the angular layout of measurement points on the predictor $\operatorname{GoF}\left(s_{\max }=25, R=600 \mathrm{~m}\right)$.

in a triple array of points. The widest measurement array is located along the circle of radius $300 \mathrm{~m}$, with the prediction point as its center. It is also assumed that the wave elevation can be measured in real time at the prediction location and included in the set of measurements.

The computation time to obtain the prediction matrix was of the order of 5-10 min using a 3.50-GHz, 8-core Intel processor, which is still compatible for an update rate consistent with the evolution of sea conditions. The GoF obtained with the proposed triple measurement layout is shown in Fig. 16. A prediction accuracy higher than $90 \%$ seems achievable up to approximately $50 \mathrm{~s}$ into the future.

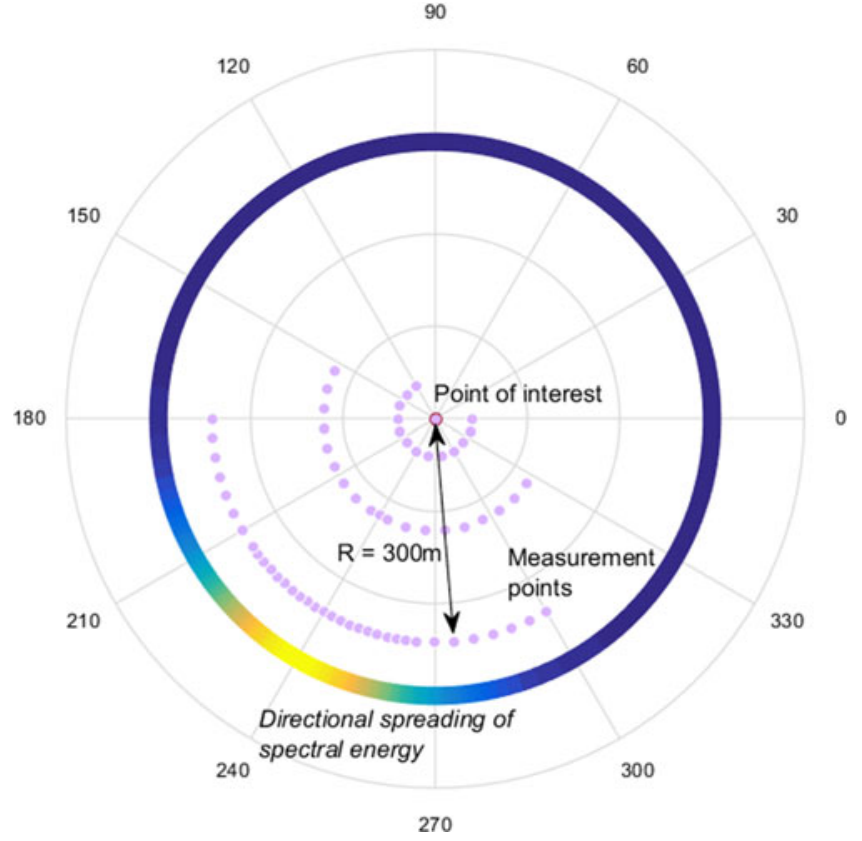

Fig. 15. Triple angular layout.

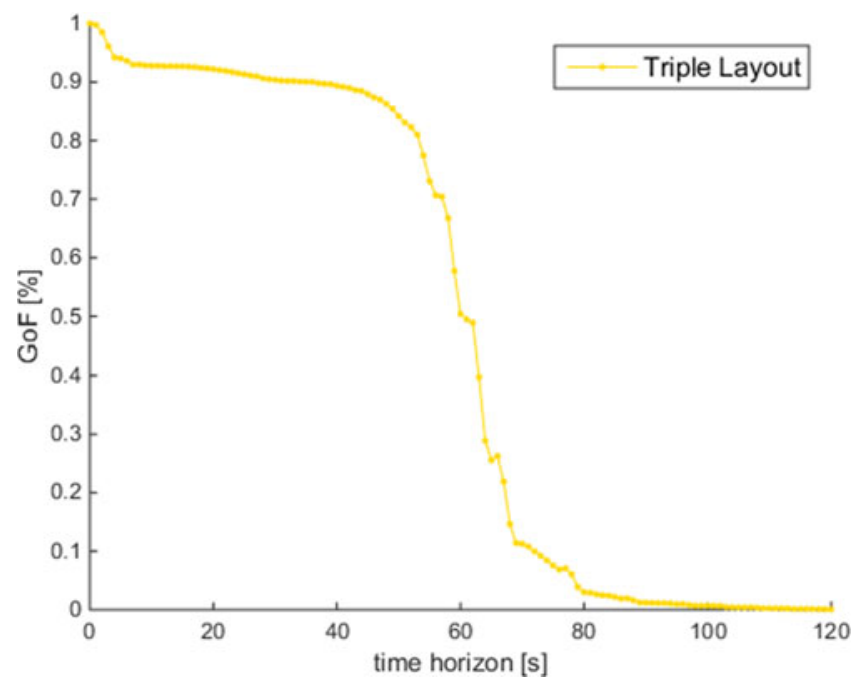

Fig. 16. GoF obtained from the triple angular layout using the last measured $250 \mathrm{~s}\left(s_{\max }=25\right)$.

\section{CONCLUSION AND DISCUSSIONS}

\section{A. Theoretical Results}

Assuming perfect knowledge of the wave field statistical characterization, in the form of a directional spectrum, the measurement and prediction values can be treated as a multidimensional Gaussian vector with a known variance-covariance matrix. For any combination of measurement and prediction points in time and space, the optimal prediction law can thus be easily derived. The prediction vector is obtained from the measurement vector through multiplication by a prediction matrix. Without requiring any numerical simulation, the prediction error can be 
immediately characterized as a Gaussian vector with a known variance-covariance matrix.

The proposed Gaussian formalism encompasses both "looking backward" (when prediction and measurement points have the same location) and "looking forward" approaches (when measurements are taken at some distance from the prediction location).

Regarding the "looking backward" approach, a few numerical examples based on the favorable case of a sharply concentrated, unimodal wave spectrum suggest that no reasonably accurate forecasts can be achieved as little as one typical wave period into the future. However, other physical quantities, such as the wave excitation forces acting on a mechanical structure, could perhaps lend themselves better prediction, provided they can be duly measured or estimated.

If the wave field can be described as unidirectional, deterministic forecasts can be achieved up to long time horizons (more than 2 min) by using measurements at only one up-wave location, over a sufficiently long period of time. Using the spectrumbased predictor, the deterministic predictable zone is found to be a function of the lowest and largest group speeds present in the wave spectrum, which corroborates the results of previous works [3], [12].

However, actual wave fields cannot be realistically assumed to be unidirectional and must rather be described by means of a multidirectional wave spectrum. Wave directionality makes the prediction task significantly more complex, involving measurements at numerous locations to achieve reasonably accurate forecasts.

Many more possibilities of measurement layouts, than those shown in this paper, could be investigated. The proposed theoretical framework will help in assessing the performance of various measurement configurations. The required time horizon and prediction accuracy depend on the practical application considered.

\section{B. Practical Applicability}

In this section, the applicability of the spectrum-based predictor to real-time wave forecasting is discussed. Several benefits can be expected, which are as follows.

1) The forecasts provided are statistically consistent, in the sense that they fade out to zero for large time horizons and distances.

2) The prediction task itself is a simple matrix multiplication, which is not computationally demanding. The prediction matrix must only be updated at regular time intervals, consistent with the rate at which the wave conditions evolve (e.g., every $30 \mathrm{~min}$ ). The computational burden is thus shifted from the prediction itself to a "preprocessing" phase, which consists of computing the prediction matrix.

3) For a given combination of measurement points, the predictor is optimal in a least mean square error sense, at least within spatial and temporal scales compatible with the stationarity and homogeneity of the wave process.
4) The wave spectrum, upon which the proposed predictor is based, reflects physical processes (wave development under the effect of wind, propagation, diffraction, etc.) that can be, to some extent, understood and predicted by means of physical representations. Sea state predictions are typically implemented through numerical models, which describe the physics of wave development and propagation via an energy balance equation, numerically solved across a geographical grid (see, for example, [16]). Such models can allow for real-time wave spectrum estimation and/or forecast in the geographical area of interest. The resulting estimated and/or forecast spectra can then be used to compute the prediction matrix. Local measurements, if available, can complement or replace the physical predictions. Thus, the statistical information, corresponding to the physical processes of wave generation and propagation, can be incorporated in the spectrum-based predictor, whereas it is completely ignored in other DSWP techniques.

However, considerable challenges remain, which are as follows.

1) As with other prediction approaches, the availability of measurements surrounding the point of interest is crucial. Measurements could be carried out using radars or wave buoys. In the former case, many measurement locations are available at the same time; however, the achievable forecast quality will ultimately depend on the spatial and temporal resolution of the radar. $X$-band radars seem to be the most suitable technological choice (see, for example, [17]). Irrespective of the technological choice, the possible presence of measurement noise must be taken into account.

2) Estimates and/or forecasts of the directional spectrum in the geographical area of interest need to be available in real time and updated at a rate matching the evolution of wave conditions. To this end, the use of both local measurements and outputs from meteorological models could be considered. The sensitivity of the predictor performance to the accuracy of spectrum estimates should be investigated. In particular, the properties of the technique in nonstationary seas deserve attention.

3) The prediction matrix computation must be carried out within a reasonable amount of time, which can be a challenging task if the number of measurement points is large; indeed, the covariance for each pair of points must be computed, and the eigenvalue decomposition of the covariance matrix must be carried out (see Section III). In the heaviest numerical example of Section VI, involving a triple array of measurement locations (see Fig. 15) and $250 \mathrm{~s}$ of past values, the construction of the prediction matrix took between 5 and 10 min using a $3.50-\mathrm{GHz}, 8$-core Intel processor. This task, however, must be performed only once every time the spectrum estimate is updated, for example, every $30 \mathrm{~min}$. If the spectrum evolution can be forecast to some extent, the construction of the prediction matrix can even be carried out in advance. 
4) In relation to the previous point, the covariance matrix may be subject to numerical issues. In fact, if measurements at all locations are taken at the same set of sampling instants, it is easily shown that the covariance matrix is Hermitian, positive definite block Toeplitz. Such matrices can become ill-conditioned for large orders [18]. This issue, although not significant for the cases considered in this paper, is currently under close study by the authors.

5) Finally, optimization schemes could be investigated, to determine an ideal set of measurement points, ensuring accurate predictions while being computationally tractable.

\section{Comparison With DSWP Methods}

The proposed predictor takes advantage of statistical information, to which DSWP techniques are blind. However, as stressed in Section VII-B, the efficient computation of the prediction matrix is a crucial question, especially in comparison with other DSWP techniques, which do not require such heavy pre-computation. DSWP, which involves the identification and propagation of a number of wave components, can handle a large number of measurement points, through efficient methods, such as 2-D fast Fourier transform (FFT) [3], 3-D FFT [19], [20], or other signal processing tools [1], [17]. In contrast, the construction and eigenvalue decomposition of the variance-covariance matrix, corresponding to the same amount of measurements, could be computationally intractable within a sufficiently small time window.

Therefore, it should be assessed whether a smaller number of optimally used measurements can be preferable to a larger set of measurements, exploited in a suboptimal way. This question is analogous to the comparison, presented in [3], between the so-called "multiple fixed points" method, which only uses a few well-chosen measurement locations within the radar range, with "2-D linear DSWP," whereby measurements in the entire surface covered by the radar are processed.

Another significant difference with respect to DSWP techniques lies in the data assimilation process. In a typical DSWP implementation, given wave data recorded within a certain space or time interval, the sinusoidal functions, describing the propagation of individual wave components, must be identified. However, the wave signal is nonperiodic over the finite time or space support considered, which can induce spectral leakage, and thus errors in the estimation of the Fourier coefficients of the propagating waves. To circumvent the issue of nonperiodicity, various techniques are studied in [1]. Traditional signal processing windowing functions are found to distort the spectral content of the measured wave, and therefore do not constitute suitable options. Alternatives to windowing functions exist, such as the end-matching technique and the socalled data extension method, whereby the recorded signal is extended so as to form a periodic signal while keeping a realistic frequency content. In [1], the latter technique shows the best performance: the deterministic prediction zone of a unidi- rectional wave field can be identified with good accuracy using simulations.

In contrast, the predictor proposed in this paper does not explicitly represent individual wave components, and therefore does not require any specific precaution at the stage of measurement assimilation. The deterministic prediction zone can still be correctly identified without resorting to any simulation.

Other limitations are not specific to the spectrum-based predictor, but rather apply to all methods based on the linear wave theory, i.e., in particular, most DSWP techniques.

1) The predictor should be used within spatial and temporal scales compatible with the assumptions of homogeneity and stationarity. For a theoretically consistent implementation of the spectrum-based predictor, studying the properties of dynamically evolving Gaussian fields [21] could be interesting.

2) The Gaussian sea assumption excludes shallow water and extreme weather conditions [8], as well as breaking, and rogue, waves.

Overall, although it is, as yet, impossible to ascertain whether the proposed formulation can provide any practical benefit for real-time implementation, the idea of incorporating available information about the current wave spectrum certainly deserves attention.

\section{REFERENCES}

[1] L. Abusedra and M. Belmont, "Prediction diagrams for deterministic sea wave prediction and the introduction of the data extension prediction method," Int. Shipbuilding Prog., vol. 58, no. 1, pp. 59-81, 2011.

[2] J. M. Giron-Sierra and S. Esteban, "The problem of quiescent period prediction for ships: A review," Proc. 8th IFAC Conf. Control Appl. Marine Syst., Rostock-Warnemünde, Germany, Sep. 15-17, 2010.

[3] M. Belmont et al., "An examination of the feasibility of linear deterministic sea wave prediction in multidirectional seas using wave profiling radar: Theory, simulation, and sea trials," J. Atmos. Ocean. Technol., vol. 31, no. 7, pp. 1601-1614, 2014.

[4] J. V. Ringwood, G. Bacelli, and F. Fusco, "Energy-maximizing control of wave-energy converters: The development of control system technology to optimize their operation," IEEE Control Syst., vol. 34, no. 5, pp. 30-55, Oct. 2014.

[5] J. Falnes, Ocean Waves and Oscillating Systems: Linear Interactions Including Wave-Energy Extraction. Cambridge U.K.: Cambridge Univ. Press, 2002, Ch. 6, pp. 196-224.

[6] F. Fusco and J. V. Ringwood, "A study of the prediction requirements in real-time control of wave energy converters," IEEE Trans. Sustain. Energy, vol. 3, no. 1, pp. 176-184, Jan. 2012.

[7] F. Fusco and J. V. Ringwood, "Short-term wave forecasting for real-time control of wave energy converters," IEEE Trans. Sustain. Energy, vol. 1, no. 2, pp. 99-106, Jul. 2010.

[8] M. K. Ochi, Ocean Waves: The Stochastic Approach. Cambridge U.K.: Cambridge Univ. Press, 2005.

[9] B. S. Connell, J. P. Rudzinsky, C. S. Brundick, W. M. Milewski, J. G. Kusters, and G. Farquharson, "Development of an environmental and ship motion forecasting system," in Proc. ASME 34th Int. Conf. Ocean, Offshore, Arctic Eng., St. John's, NL, Canada, May 31 to June 5, 2015, Paper OMAE2015-42422.

[10] K. Hasselmann et al., Measurements of Wind-Wave Growth and Swell Decay During the Joint North Sea Wave Project (JONSWAP). Hamburg, Germany: Deutsches Hydrographisches Institut, 1973, pp. 1-95.

[11] A. Mérigaud and J. V. Ringwood, "Free-surface time-series generation for wave energy applications," IEEE J. Ocean. Eng., vol. 43, no. 1, pp. 19-35, Jan. 2018.

[12] P. Naaijen, K. Trulsen, and E. Blondel-Couprie, "Limits to the extent of the spatio-temporal domain for deterministic wave prediction," Int. Shipbuilding Prog., vol. 61, no. 3-4, pp. 203-223, 2014. 
[13] J. G. de Boer, "On the correlation functions in time and space of windgenerated ocean waves," SACLANTCEN, Sound Propagation Group, SACLANT ASW Res. Centre, La Spezia, Italy, Tech. Rep. 160, 1969.

[14] Y. Goda, "Overview on the applications of random wave concept in coastal engineering," Proc. Jpn. Acad., B, vol. 84, no. 9, pp. 374-385, 2008.

[15] M. L. Eaton, Multivariate Statistics: A Vector Space Approach (Lecture Notes-Monograph Series), vol. 53. Hoboken, NJ, USA: Wiley, 2007, pp. 1-512. [Online]. Available: http://www.jstor.org/stable/20461449

[16] S. Gallagher, R. Tiron, E. Whelan, E. Gleeson, F. Dias, and R. McGrath, "The nearshore wind and wave energy potential of Ireland: A high resolution assessment of availability and accessibility," Renewable Energy, vol. 88, pp. 494-516, 2016.

[17] T. Hilmer and E. Thornhill, "Observations of predictive skill for realtime deterministic sea waves from the WaMoS II," in Proc. MTS/IEEE OCEANS, Washington, DC, USA, 19-22 Oct. 2015, pp. 1-7.

[18] S. Serra, "Spectral and computational analysis of block Toeplitz matrices having nonnegative definite matrix-valued generating functions," BIT Numer. Math., vol. 39, no. 1, pp. 152-175, 1999.

[19] P. Naaijen and E. Blondel-Couprie, "Reconstruction and prediction of short-crested seas based on the application of a 3D-FFT on synthetic waves. Part 1: Reconstruction," in Proc. 31st Int. Conf. Ocean, Offshore Arctic Eng., Rio de Janeiro, Brazil, Jul. 1-6, 2012, pp. 43-53.

[20] E. Blondel-Couprie and P. Naaijen, "Reconstruction and prediction of short-crested seas based on the application of a 3D-FFT on synthetic waves. Part 2: Prediction," in Proc. ASME 31st Int. Conf. Ocean, Offshore, Arctic Eng., Rio de Janeiro, Brazil, Jul. 1-6, 2012, pp. $1-16$.

[21] A. Baxevani, K. Podgórski, and I. Rychlik, "Dynamically evolving Gaussian spatial fields," Extremes, vol. 14, no. 2, pp. 223-251, 2011.

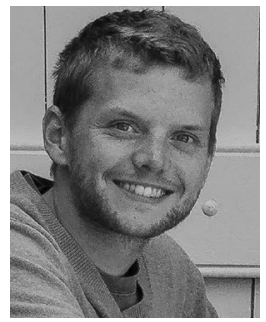

Alexis Mérigaud received the Engineering degree in energy economics from ENSTA, Paris, France, in 2012. He has been working toward the Ph.D. degree at the Centre for Ocean Energy Research, Maynooth University, Maynooth, Ireland, since January 2015.

At ENSTA, he specialized in energy management and economics. He then studied public administration for two years at Sciences Po Paris. His previous experience in the field of energy includes economic modeling, as well as technical regulatory aspects, of energy networks and markets. He is currently working on energy forecasting and predictive maintenance applied to marine renewable energy sources.

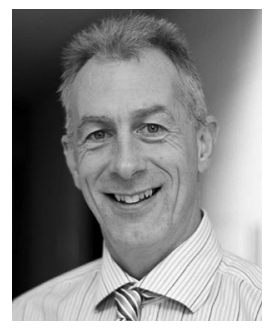

John V. Ringwood (M'87-SM'97) received the Diploma in electrical engineering from Dublin Institute of Technology, Dublin, Ireland, in 1981 and the $\mathrm{Ph} . \mathrm{D}$. degree in control systems from Strathclyde University, Glasgow, Scotland, in 1985.

$\mathrm{He}$ is currently a Professor in Electronic Engineering at Maynooth University, Maynooth, Ireland, and the Director of the Centre for Ocean Energy Research, Maynooth University. From 2000 to 2005, he was the Head of the Electronic Engineering Department, Maynooth University, National University of Ireland Maynooth, developing the department from a greenfield site. His research interests include time-series modeling, wave energy, control of plasma processes, and biomedical engineering.

Dr. Ringwood is a Chartered Engineer and a Fellow of the Institution of Engineers of Ireland. 